\title{
Substance Abuse among Middle Eastern Immigrants and Refugees in Greater Detroit, Michigan, U.S.
}

\author{
Hikmet J Jamil, MD, PhD ${ }^{1^{*}}$, Ayesha Niazy, MD ${ }^{1}$, Mada H Jamil, MD, MPH and Salman Rawaf, MD, PhD \\ ${ }^{1}$ Department of Family Medicine \& Public Health Science, School of Medicine, Wayne State University, USA \\ ${ }^{2}$ St. John Providence Physician Network, Sterling Heights, USA \\ ${ }^{3}$ Department of Primary Care and Public Health, School of Public Health, Imperial College, UK
}

\begin{abstract}
Background: Substance usage is a prevailing endemic around the globe. It has a global effect on the economic and social aspects of society, making it crucial to assess risk factors and prevalence. However, a large number of immigrants and refugees who came to the U.S., have come from Middle Eastern countries in conflict with consequent psychiatric disorders like depression and stress. Literature shows that over $9.2 \%$ of the U.S. population alone is involved in illicit drugs and $22.7 \%$ is involved in alcohol use. Our study's objective was to assess the prevalence and risk factors of substance use (Alcohol and illicit drugs) among immigrants and refugees in Greater Detroit area of state of Michigan, U.S.
\end{abstract}

Methods: A 7.5\% random sample from an Iraqi address list of $5555(n=350)$ (immigrants $(n=152)$, refugees $(n=198)$ ) residing in Greater Detroit was studied. We analyzed alcohol, street drugs, amphetamine and sedative usage in this population via binary logistic regression, linear regression, and Chi square analysis.

Results: Results indicate that there was a significant difference in prevalence of substance usage between immigrants and refugees, with the latter having a higher street drug use $(p<0.001)$. Immigrants have a higher alcohol use $(43.4 \%)(p$ $<0.001$ ). The predictors for drinking alcohol were: male, smokers and those without health insurance. Depression was a predictor for using any substance drugs. People who use substances have higher incidence of chronic headaches and lumbago $(p<0.001)$.

Conclusion: Overall, prevalence of substance usage is higher amongst refugees as compared to their immigrant counterparts. Binging alcohol was higher among immigrants (43.4\%). The predictors for using one or more substances included male gender and those with associated smoking.

Keywords

Iraqis, Substance use risk factors, Chronic diseases, Self-rated health

\section{Introduction}

In general, a refugee is defined as 'person who has been forced to leave their country in order to escape war, persecution, or natural disaster'. Whereas an immigrant is 'a person who comes to live permanently in a foreign country'. In Michigan the number of immigrant comprise $6.4 \%$ of the state's population [1], and with the number of refugees who have immigrated to U.S. in 2016, 5\% of those refugees chose Michigan as their new home [2]. The majority of these refugees and immigrants who have relocated to the U.S. have come from the wars that have torn Middle Eastern countries. The diverse background and hard circumstances bring with it psychiatric disorders like depression and stress $[3,4]$. Along with such issues comes the obstacle of adapting to a new country and overcoming problems such as cultural acceptance, language, and discrimination [5]. The combination of psychiatric disorders and stress of settling in to a new country makes immigrants and refugees vulnerable to substance use $[3,6]$. Substance abuse (alcohol and illicit drugs) is endemic in today's world. Over $9.2 \%$ of the U.S. population alone is involved in illicit drugs and $22.7 \%$ is involved in alcohol use [7]. This in turn has cost over $\$ 700$ billion a year in crime, lost work productivity, and healthcare - all in. These current statistics of sub-

*Corresponding author: Hikmet J Jamil, Department of Family Medicine \& Public Health Science, School of Medicine,Wayne State University, Michigan, USA

Accepted: July 11, 2019

Published online: July 13, 2019

Citation: Jamil HJ, Niazy A, Jamil MH, et al. (2019) Substance Abuse among Middle Eastern Immigrants and Refugees in Greater Detroit, Michigan, U.S.. Adv Environ Stud 3(1):209-215 
Citation: Jamil HJ, Niazy A, Jamil MH, et al. (2019) Substance Abuse among Middle Eastern Immigrants and Refugees in Greater Detroit, Michigan, U.S.. Adv Environ Stud 3(1):209-215

stance abuse in U.S. show not only that there is a high prevalence, but that it has become critical to learn more about the reasons behind these numbers and how to combat them. Statistics amongst the Middle Eastern population of Michigan show that the prevalence of substance abuse is as follows: alcohol $45.6 \%$, marijuana street drug $1.5 \%$, and prescription $0.6 \%[8,9]$. These numbers further pique our interest to dwell into the reason among the sub populations (immigrant's vs. refugees) of substance users in the Arab-American community of Michigan. Comparing the two groups - immigrants vs. refugees-, the latter face more direct challenges. Refugees get the shorter end of the stick as they face more strenuous conditions in their home country including starvation, abduction, and violence [10], which has forced them to seek asylum in their new adopted country. Substance abuse is also an issue in Middle Eastern countries, despite the religious stance on such a topic, which forbids the use of alcohol and illicit drugs [11]. Hence, it is more common in the second generation of immigrants as compared to their predecessors $[12,13]$. The reason can be related to the social and cultural adaptability of the second-generation of immigrants to the new country $[12,13]$. This can also be straying from their roots and the culture of their origin country, which the first generation of immigrants try to keep intact by living in areas that comprise same-origin immigrants and refugees [14,15]. Despite this, the level of substance abuse amongst immigrants and refugees is still lower compared to native-born Americans. This phenomenon is called 'immigrant paradox' [13]. Reasons for drinking alcohol amongst immigrants and refugees of U.S. vary from social acceptance [16], self-treatment of issues like stress, pain, insomnia [16], to forget their past [17]. Arab-American immigrants and refugees have a lower use of non-prescription drugs and marijuana, compared to U.S. born non-Hispanic; but not surprisingly have similar levels of use when compared to other immigrants [18]. Substance abuse has evident gender differences amongst immigrants and refugees [19]. Women are $51 \%$ more likely to abstain from alcohol as compared to their male counterparts (6\%) [19]. The latter are also more likely to be moderate to heavy drinkers (39\% vs. only $6 \%$ of females) [19]. Immigrant, refugee, and U.S. born men are more likely to have a positive correlation with underlying depression and alcohol use compared to females [20]. Married individuals have a lower prevalence of substance abuse [21]. This was also evident in individuals who changed their marital status from single to married, decreasing their alcohol use [22]. Companionship brings mental satisfaction and gratitude that have positive effects on the mental health of individuals and discourages them from damaging habits like substance abuse [23]. Educated individuals have a lower prevalence of substance abuse, e.g. drinking alcohol and illicit drug use [24]. Education allows individuals to gain appropriate knowledge on the detrimental effects of abusing substances [25]. It also helps in having a focus in life and allowing them to aim for achievements in life that would not be possible had they been involved in substance abuse [25]. Although tobacco usage is not perceived as a problematic drug, data suggests that those involved in tobacco usage are also involved in illicit drug use and alcohol consumption [26]. Tobacco use is also connected to mental health problems [26]. Individuals suffering from mental health issues, like anxiety and depression, have a correlation with tobacco usage and hence, also with abusing substances [26]. Mental health problems also have a direct correlation with substance abuse in veterans [27]. Immigrants and refugees who are also veterans have all the high-risk factors associated with illicit drug use and alcohol usage [27]. We mentioned some data on substance abuse in Arab-Americans but, there was no data on particular substance abuse or on the general health among Iraqi American (Immigrants/Refugees), for that reason, a health assessment study among Iraqi Americans was conducted. Despite having immigrated to U.S. from the same country (Iraq) sharing similar cultural background, language, habit, and religion they differ according to the immigration status.

In this study, we aim to show the prevalence and assess risk factors of different substance abuse (Alcohol and illicit drugs) in two groups, comparing their legal status - immigrants and refugees- in the Greater Detroit area of the state of Michigan, U.S. We hypothesize that the prevalence of substance abuse will be higher among refugees compared to immigrants If this is true, that would mean the refugees were more exposed to trauma in Iraq (chemical and non-chemical - psychological stressors) and to different types of trauma (employment status, discrimination, lack of language etc.) at the host country (U.S.).

\section{Methods}

\section{Participants}

We conducted a survey in 2004 amongst the Iraqi American population of the Greater Detroit area in Michigan, U.S. The study participants were approached via media outlets - TV, radio etc. in the localities where the majority of Iraqi Americans reside. The survey conducting team included research assistants (retired physicians) who spoke Arabic, which is the same language spoken by the study participants. They were also familiar with the participants' culture. This allowed us to have a more open and honest survey because the team had the advantage of taking the participants into confidence easily due to affinity. Hiring retired physicians also played a big role in achieving, what we perceive, as unbiased and honest answers to the survey, as study participants are generally more comfortable and honest when the interviewee is a physician. At the end of the survey 5,490 addresses were collected. They were then coded and entered into SPSS. A sample of $7.5 \%$ (411 addresses) was randomly selected, because of limited funding. Forty-four of these were excluded because they did not meet the criteria (e.g. not born in Iraq or had moved at the time of research visit). Out of the 367 eligible candidates 17 declined to participate, for reasons including lack of time, lack of interest in the study or no reason mentioned at all. Therefore, our final study group was of 350 participants by 2005 [25]. The target of the survey was to assess the health conditions of the Iraqi population. Within the survey questionnaires, questions related to the substance 
Citation: Jamil HJ, Niazy A, Jamil MH, et al. (2019) Substance Abuse among Middle Eastern Immigrants and Refugees in Greater Detroit, Michigan, U.S.. Adv Environ Stud 3(1):209-215

abuse (Alcohol and illicit drugs) were present, allowing the research team to conduct this study.

\section{Measures}

The structured questionnaire used in the interview process was a validated instrument used in large-scale surveys [28]. 108 questions covering different aspects of participants' health and background were in the survey questionnaire. Questions included information about their demographics, immigration, health problems, and substance abuse. We classified the study population according to their legal status: immigrants $(n=152)$ and refugees $(n=198)$. For substance abuse, there were 9 questions for alcohol, and one question for each of the illicit drugs as follows: for sedative drugs, the question was "Use sedatives, tranquilizers, or pain killers for non-medical reasons"; for stimulant drugs, the question was "Use amphetamines or inhalants such as glue or aerosol sprays"; for street drugs, the question was "Use any other illegal or street drugs such as cocaine, crack, hallucinogens"; and the last question was for marijuana or hash. Since there were no replies to marijuana use, it was not included in the study. For the illicit drugs, the participant had two options to respond; either "yes" or "no" for each question. We established some other variables, e.g. Illicit drugs which included the three drugs (sedative + stimulant + street drugs). In the 9 alcohol questions the participant had two options to respond- either (1) Yes or (2) No. Examples of such questions are; 'During the last month have you drunk alcohol?', 'Have you thought you should cut down on your drinking of alcohol' etc. Then, for alcohol, we combined the nine questions and then established a variable with two categories (never drink alcohol or drink alcohol in his/her life); another new variable was created which included both illicit drugs and alcohol and was named 'substance abuse'. Some chronic health problems could increase the risk for an individual to use illicit drugs or even alcohol; such as depression, headache, sleep apnea, lumbago, arthritis and musculoskeletal disorders. These chronic health questions were asked in the survey and were considered predicting risk factors for substance abuse. We established a new variable for depression based on two sets of questions in the survey; one set consisted of four questions; the participant had to select one of the four options for each question. The options were (1) A little bit, (2) Moderately, (3) Quite a bit, (4) Extremely. The second set consisted of six questions and the participants had to answer either (1) Yes or (2) No to each. Combing the ten questions led to a scale ranging from 0 to 22 . The depression scale outcome was as follows: 0-1 meant no depression, 2-4 symptoms meant minor depression and $5+$ symptoms meant major depression. Iraqis who settled either as immigrant or refugees were exposed to trauma either from wars or from the dictatorship government. These aspects were considered when participants answered questions on environmental exposure (Chemical and non-chemical or stressor) as predicting risk factors for substance abuse. Non-Chemical or stressors were assessed in the population by asking 8 questions (e.g. witnessed anyone dying, been under small arms fire and exposure to dead bodies), participants had to select one of the three options for each question, the options were; (1) Expose to $<5$ day, (2) 5-30 days and (3) 31+ days. The range of stressor scale was between 0 and 24 . The chemical environmental exposure scale consisted of 15 questions including chemicals, smoke from oil well fires, and other petrochemical fuels. The range of the scale for environmental exposure was between 0 and 45 points. The environmental and stressor scales have demonstrated excellent psychometric properties with a Cronbach's alpha of $>0.85$. The participants were also asked to fill responses regarding their self-rated health (SRH). This SRH scale is shown to help predict future health and mortality in current healthy subjects [24] as shown in Table 1.

\section{Procedure/Interview process}

This study was approved by IRB [WSU/IRB/HIC\# 086903B3E].

Table 1: Prevalence of self-rated health by legal status and linear regression analysis to predict risk factors for excellent health.

\begin{tabular}{|c|c|c|c|c|c|c|}
\hline \multirow[t]{2}{*}{ Currently SRH } & \multicolumn{2}{|l|}{ Never used } & \multirow[t]{2}{*}{$\mathbf{P}$} & \multicolumn{2}{|l|}{ Substances abuse } & \multirow[t]{2}{*}{$\mathbf{P}$} \\
\hline & Refugees $(n=137)$ & Immigrants $(n=77)$ & & Refugees $(n=68)$ & Immigrants $(n=68)$ & \\
\hline Excellent & 4.4 & 16.9 & \multirow{5}{*}{$P<0.001$} & 4.4 & 11.8 & \multirow{5}{*}{$P<0.001$} \\
\hline Very good & 8 & 29.9 & & 11.8 & 33.8 & \\
\hline Good & 24.8 & 35.1 & & 30.9 & 33.8 & \\
\hline Fair & 42.3 & 16.9 & & 36.8 & 19.1 & \\
\hline Poor & 20.4 & 1.3 & & 16.2 & 1.5 & \\
\hline \multicolumn{7}{|c|}{ Linear regression to Predict risk factors for Excellent health } \\
\hline \multirow[t]{2}{*}{ Risk factors } & Standardized & \multirow[t]{2}{*}{$\mathrm{t}$} & \multirow[t]{2}{*}{ Sig } & \multicolumn{2}{|l|}{$95.0 \%$ for $B$} & Partial \\
\hline & Beta & & & Lower bound & Upper bound & \\
\hline No or Minimal depression & 0.33 & 5.73 & 0.000 & 0.06 & 0.11 & 0.30 \\
\hline Younger age & 0.14 & 3.35 & 0.001 & 0.01 & 0.03 & 0.18 \\
\hline Unemployed & -0.17 & -3.29 & 0.001 & -0.61 & -0.15 & -0.18 \\
\hline No or Minimal back disorders & 0.11 & 2.42 & 0.016 & 0.05 & 0.44 & 0.13 \\
\hline
\end{tabular}

Variables in the equation: Legal status, Age, Years in U.S., Served in the Army, Gender, Marital status, Education, Employment status, Religious status, Health insurance, Smoking status, Exposure to chemicals, Exposure to stressor, Each of chronic diseases, Depression. 
Citation: Jamil HJ, Niazy A, Jamil MH, et al. (2019) Substance Abuse among Middle Eastern Immigrants and Refugees in Greater Detroit, Michigan, U.S.. Adv Environ Stud 3(1):209-215

Participants were assured their specifications would be kept confidential and were interviewed in their native language by Arab speaking research assistants and following a structured survey that was also written in Arabic.

\section{Statistical analysis}

Different statistical analyses were used to compare and predict risk factors for each substance abuse amongst immigrants vs. refugees. These include chi-square, learner

Table 2: Percentage of study population who used or not one or more substances* by demography and legal status.

\begin{tabular}{|c|c|c|c|c|c|c|c|}
\hline \multirow[t]{3}{*}{ Variable } & \multirow[t]{3}{*}{ Sub-variable } & \multicolumn{2}{|l|}{ Never Used } & \multirow[t]{3}{*}{$\mathbf{P}^{* *}$} & \multicolumn{2}{|l|}{ Substances Used } & \multirow[t]{3}{*}{$\mathbf{P}^{* *}$} \\
\hline & & Refugees $(n=137$ ) & Immigrants $(n=77)$ & & Refugees $(n=68)$ & Immigrants $(n=68)$ & \\
\hline & & M (SD) & $M(S D)$ & & M (SD) & M (SD) & \\
\hline \multicolumn{2}{|l|}{ Age in year } & $44.5(7.5)$ & $45.4(7.0)$ & n.s & $45.4(8.2)$ & $48.0(6.8)$ & \multirow[t]{2}{*}{0.01} \\
\hline \multicolumn{2}{|l|}{ Years In U.S. } & $8.3(3.0)$ & $24.9(7.1)$ & 0.001 & $8.5(3.5)$ & $26.6(6.8)$ & \\
\hline \multicolumn{2}{|l|}{ Chemical exposures } & $4.0(4.9)$ & $0.1(0.5$ & 0.001 & $4.4(5.8)$ & $0.4(1.5)$ & 0.001 \\
\hline \multicolumn{2}{|l|}{ Stressor exposures } & $8.0(5.5)$ & $0.3(1.5)$ & 0.001 & $8.1(6.5)$ & $0.6(2.5)$ & 0.001 \\
\hline & & $\%$ & $\%$ & & $\%$ & $\%$ & \\
\hline \multirow[t]{2}{*}{ Served in Iraqi army } & No & 67.2 & 92.2 & 0.001 & 39.7 & 73.5 & \multirow[t]{2}{*}{0.001} \\
\hline & Yes & 32.8 & 7.8 & 0.001 & 60.3 & 26.5 & \\
\hline \multirow[t]{2}{*}{ Gender } & Female & 59.1 & 54.5 & \multirow[t]{2}{*}{ n.s } & 26.5 & 19.1 & \multirow[t]{2}{*}{ n.s } \\
\hline & Male & 40.9 & 45.5 & & 73.5 & 80.9 & \\
\hline \multirow[t]{2}{*}{ Marital status } & All others & 15.3 & 27.3 & \multirow[t]{2}{*}{0.01} & 10.3 & 30.9 & 0.01 \\
\hline & Married & 84.7 & 72.7 & & 89.7 & 69.1 & \multirow{3}{*}{ n.s } \\
\hline \multirow[t]{2}{*}{ Education level } & $<$ H.S. & 43.1 & 33.8 & \multirow[t]{2}{*}{ n.s } & 47.1 & 32.4 & \\
\hline & H.S. + & 56.9 & 66.2 & & 52.9 & 67.6 & \\
\hline \multirow[t]{2}{*}{ Work status } & No & 63.5 & 32.5 & 0.001 & 44.1 & 14.9 & 0.001 \\
\hline & Yes & 36.5 & 67.5 & 0.001 & 55.9 & 85.1 & 0.001 \\
\hline \multirow[t]{2}{*}{ Health insurance } & No & 21.2 & 40.3 & 0.001 & 29.4 & 48.5 & 0.001 \\
\hline & Yes & 78.8 & 59.7 & 0.001 & 70.6 & 51.5 & 0.001 \\
\hline \multirow[t]{2}{*}{ Smoke tobacco } & No & 79.6 & 75.3 & \multirow[t]{2}{*}{ n.s } & 50 & 47.1 & \multirow[t]{2}{*}{ n.s } \\
\hline & Yes & 20.4 & 24.7 & & 50 & 52.9 & \\
\hline
\end{tabular}

*Substance usage studied includes: Alcohol, Street drugs, Sedatives, Stimulants; ${ }^{* *} \mathrm{P}$ n.s $=$ Not significant.

Table 3: Percentage of chronic medical condition by substance abuse* and by legal status of study population with test of significance.

\begin{tabular}{|c|c|c|c|c|c|c|c|}
\hline \multirow[t]{2}{*}{ Medical condition } & \multirow{2}{*}{$\begin{array}{l}\text { No } \\
\text { Yes }\end{array}$} & \multicolumn{2}{|l|}{ Never Used } & \multicolumn{2}{|c|}{ Substances Used } & \multirow[b]{2}{*}{ Immigrants $(n=68)$} & \multirow[b]{2}{*}{$\mathbf{P}^{*}$} \\
\hline & & Refugees $(n=137)$ & Immigrants $(n=77)$ & $\mathbf{P}^{*}$ & Refugees $(n=68)$ & & \\
\hline \multirow[t]{2}{*}{ Recurrent headaches } & No & 62.8 & 90.9 & 0.001 & 70.6 & 97.1 & 0.001 \\
\hline & Yes & 37.2 & 9.1 & 0.001 & 29.4 & 2.9 & 0.001 \\
\hline \multirow[t]{2}{*}{ Sleep apnea or Narcolepsy } & No & 46 & 68.8 & 0.001 & 47.1 & 61.8 & \multirow[t]{2}{*}{ n.s } \\
\hline & Yes & 54 & 31.2 & 0.001 & 52.9 & 38.2 & \\
\hline \multirow[t]{2}{*}{ Arthritis or Rheumatism } & No & 73.7 & 85.7 & \multirow[t]{2}{*}{0.01} & 80.9 & 85.3 & \multirow[t]{2}{*}{ n.s } \\
\hline & Yes & 26.3 & 14.3 & & 19.1 & 14.7 & \\
\hline \multirow[t]{2}{*}{ Lumbago (back disorder) } & No & 46 & 58.4 & \multirow[t]{2}{*}{0.05} & 60.3 & 58.8 & \multirow[t]{2}{*}{ n.s } \\
\hline & Yes & 54 & 41.6 & & 39.7 & 41.2 & \\
\hline \multirow[t]{2}{*}{ Muscles or Tendons disorders } & No & 54 & 76.6 & 0.001 & 64.7 & 79.4 & \multirow[t]{2}{*}{0.05} \\
\hline & Yes & 46 & 23.4 & 0.001 & 35.3 & 20.6 & \\
\hline \multirow[t]{3}{*}{ Depression status } & No & 18.2 & 46.8 & 0.001 & 31 & 52 & 0.001 \\
\hline & Minor & 13.1 & 27.3 & 0.001 & 10 & 31 & 0.001 \\
\hline & Major & 68.6 & 26.0 & 0.001 & 59 & 18 & 0.001 \\
\hline
\end{tabular}

"Substance abuse includes: Alcohol and Elicit drugs (Street drugs, Sedative drugs and Stimulants drugs); ${ }^{*}$ n.s = Not Significant. 
Citation: Jamil HJ, Niazy A, Jamil MH, et al. (2019) Substance Abuse among Middle Eastern Immigrants and Refugees in Greater Detroit, Michigan, U.S.. Adv Environ Stud 3(1):209-215

regression analysis, and logistic regression analysis using SPSS version 22.

\section{Results}

Based on the random sample of the study group there were significant differences between immigrant and refugee in all demographic variables as well as in chemical and non-chemical (stressors) exposure. Table 2 shows the prevalence of substance abuse (those with substance abuse and those without) by legal status, and different demographic variables. Results indicate a significant difference between refugees versus immigrant who were on substance abuse (alcohol and illicit drugs) in all variables except gender, education and smoking tobacco. Table 3 shows refugees have

Table 4: Prevalence of substance abuse by legal status \& Test of significance.

\begin{tabular}{|c|c|c|c|}
\hline \multirow[t]{3}{*}{ Substance Used } & Refugees & Immigrants & Total $(\mathrm{n}=$ \\
\hline & $(n=205)$ & $(n=145)$ & 350) \\
\hline & No. () & No. () & No. () \\
\hline Drink Alcohol on his/her life ${ }^{* * *}$ & $46(22.4)$ & $63(43.4)$ & $109(31.1)$ \\
\hline 1- Street drugs ${ }^{* * *}$ & $20(9.8)$ & $2(1.4)$ & $22(6.3)$ \\
\hline 2- Sedatives drugs & $13(6.3)$ & $5(3.4)$ & $18(5.1)$ \\
\hline 3- Stimulants drugs & $6(2.9)$ & $2(1.4)$ & $8(2.3)$ \\
\hline Used one or more of the substance Abuse ${ }^{* *}$ & $68(33.2)$ & $68(46.9)$ & $136(38.9)$ \\
\hline \multicolumn{4}{|l|}{ Frequency substance used ${ }^{* * *}$} \\
\hline 0 & $137(66.8)$ & $77(53.1)$ & $214(61.1)$ \\
\hline 1 & $56(27.3)$ & $64(44.1)$ & $120(34.3)$ \\
\hline 2 & $7(3.4)$ & $4(2.8)$ & $11(3.1)$ \\
\hline 3 & $5(2.4)$ & & $5(1.4)$ \\
\hline
\end{tabular}

1-Street drugs (Cocaine, Crack, Hallucinogens, Heroin, or Narcotics), 2- Sedatives (Any sedatives, Tranquilizers, or Pain killers for non-medical reasons) and 3- Stimulants drugs (Amphetamines or Inhalants such as Glue or Aerosol sprays). ${ }^{* *} \mathrm{P}<0.01 ;{ }^{* * *} \mathrm{P}<0.001$.

Table 5: Logistic regression analysis to predict risk factors for each substance abuse and for all combined and illicit drugs.

\begin{tabular}{|c|c|c|c|c|c|}
\hline \multirow[t]{2}{*}{ Predicting Risk Factor } & \multirow[t]{2}{*}{ B } & \multirow[t]{2}{*}{ Sig. } & \multirow[t]{2}{*}{ OR } & \multicolumn{2}{|c|}{ 95\% C.I.for OR } \\
\hline & & & & Lower & Upper \\
\hline \multicolumn{6}{|c|}{ Drink Alcohol during his/her life } \\
\hline Christian & -1.78 & 0.000 & 0.17 & 0.08 & 13.01 \\
\hline Male & -1.72 & 0.000 & 0.18 & 0.07 & 5.25 \\
\hline Smoke tobacco & 0.94 & 0.005 & 2.56 & 1.32 & 0.82 \\
\hline recurrent headache & 1.20 & 0.037 & 3.32 & 1.08 & 3.81 \\
\hline \multicolumn{6}{|l|}{ Any Street drugs } \\
\hline Depression scale & 0.45 & 0.000 & 1.56 & 1.22 & 2.00 \\
\hline \multicolumn{6}{|l|}{ Any Sedatives drugs } \\
\hline \multicolumn{6}{|l|}{ No predictor risk factor } \\
\hline \multicolumn{6}{|l|}{ Any Stimulant drugs } \\
\hline \multicolumn{6}{|l|}{ No predictor risk factor } \\
\hline \multicolumn{6}{|c|}{ Illicit drugs (street, sedative and stimulants combined) } \\
\hline Depression scale & 0.21 & 0.003 & 1.23 & 1.07 & 1.42 \\
\hline Recurrent headaches & -1.00 & 0.042 & 0.37 & 0.14 & 0.97 \\
\hline \multicolumn{6}{|c|}{ Used one or more of the substance abuse (Elicit and Alcohol) } \\
\hline Christian & -1.36 & 0.000 & 0.26 & 0.13 & 0.51 \\
\hline Male & -1.44 & 0.000 & 0.24 & 0.11 & 0.50 \\
\hline Smoke tobacco & 0.85 & 0.004 & 2.35 & 1.32 & 4.19 \\
\hline
\end{tabular}

Note: Each of the six logistic regression analysis was including the following variables: Age, Gender, Marital status, Occupation, Health insurance, Religion, Legal status of study population, Smoking status, Chemical exposure, Non-chemical (stressor), Depression score, Each of the Six chronic diseases, Years in USA, Years in Iraqi military services. 
Citation: Jamil HJ, Niazy A, Jamil MH, et al. (2019) Substance Abuse among Middle Eastern Immigrants and Refugees in Greater Detroit, Michigan, U.S.. Adv Environ Stud 3(1):209-215

a significant prevalence in all chronic conditions except lumbago, among those who were on substance abuse, while scant differences existed only in participants with associated depression, recurrent headache and muscular/tendon disorders. It is of interest to see significant difference between refugees versus immigrant's in relation to drinking alcohol (43.4\% in immigrant's vs. $22.4 \%$ in refugees), while in all illicit drugs (street, sedative and stimulants) refugees have higher prevalence rate than immigrants, although no significant difference between the two groups except for street drugs. It is also of notice to find that the prevalence of substance abuse was higher (46.9\% vs. $33.2 \%$ ) among refugees (Table 4). However, Table 4 shows that the percentage of those who use one or more substances was higher among immigrants (46.9\%) compared to refugees (33.2\%), In the case of illicit drugs alone, refugees which are mainly Muslim have the higher prevalence $(19 \%)$ compared to immigrants which are mainly Christian (6.2\%). Table 5 predicts the risk factor of each of the substance abused by applying logistic regression analysis to each of them. Male, Christian, smokers, and those with recurrent headaches were predictors for drinking alcohol; while depression was a predictor for using street drugs, while we found no predictor for sedative and stimulant drug. However, the predictor risk factors for combined illicit drugs were depression and recurrent headache, but for the overall substance abuse the predictor risk factors were Christian (represent immigrants mainly), male and smokers. Table 1 represents the differences between study group and reported self-rated health (SRH), which indicate a significant difference between refuge vs. immigrant irrespective if they were on substance abuse or not. It also shows the predictor risk factors for excellent health through applying linear regression analysis. The predictors for excellent health included those without or minimal depression, younger age individuals, unemployed and those with none or minimal back disorders.

\section{Discussion}

The results confirm our hypothesis where the refugees have a higher prevalence rate in illicit drug use (street, sedative, stimulants), but not alcohol (43.4\% in immigrants vs. $22.4 \%$ in refugees). This could be explained by the fact that most immigrants were Christian, and they do not have any religious restriction to alcohol use as stated in Arfken's 2014 study [29].

This higher prevalence is confirmed by the relationship of circumstances that force refugees to move as stated by Russel [10], and their direct correlation with psychiatric and psychological issues agreeing with Ross and Khantizan's studies $[5,10]$.

However, there were significant differences between refugees and immigrants in most, if not all the demographic variables irrespective if they were substance abusive or not (Table 2) 33.2\% of refugees abuse one or more substances compared to $46.9 \%$ of immigrants, with (Table 4). Also, the results showed the predictable risk factors for each of the substances; where male, Christian (most of them immigrants), recurrent headache, and smoking were predictors amongst people who use alcohol; depression was a predictor for street drug users, while depression and recurrent headache were predictors among those who used illicit drugs irrespective of its kind or number. It was curious to find no predictor of legal status when testing each substance abuse alone. Our results are parallel with Padilla's paper [19], showing that males have higher prevalence of substance abuse compared to their female counterparts, also making it a risk factor for using alcohol.

Dick's 2006 [21] study shows that married individuals have a lower prevalence of substance abuse, which is also seen in our results. Being educated or being employed showed a negative correlation with substance abuse, agreeing with the findings of Johnston's study [24]. Among those who abused substances, $53 \%$ of refugees reported their health fair to poor vs. $20.6 \%$ of immigrants (as they had been longer in U.S). The predictor risk factors for excellent health are: younger age individual, unemployed, no or minimal depression and no or minimal back disorders, however, legal status was not a predictor risk factor for excellent health (Table 1).

\section{Strengths and Limitations}

The major strength of this study lies in its data collection, as it is based on a random sample. This is also the only study focusing on illegal substance usage in relation to immigration status in Iraqi American. The study had a big sample size and participants were from the same country. The study limitation is related to the survey, which was designed as a health assessment of Iraqi American in general, but authors were able to use the answers from the survey for this article.

\section{Conclusion}

The study has concluded that overall prevalence of illicit drugs was higher amongst refugees compared to their immigrant counterparts. $22.4 \%$ of refugees are involved in alcohol use. The predictors for abusing one or more substances include male gender, Christian (mainly immigrants), and those with associated smoking.

\section{Acknowledgment}

The authors appreciate the contribution of Dr Quezada Yamamoto $\mathrm{H}$ during the final stage of the publication.

\section{References}

1. American Immigration Council (2015) New americans in michigan: The political and economic power of immigrants, latinos, and asians in the great lakes state.

2. (2017) Refugee processing center.

3. Ross HE, Glaser FB, Germanson T (1988) The prevalence of psychiatric disorders in patients with alcohol and other drug problems. Arch Gen Psychiatry 45: 1023-1031.

4. Khantzian EJ (1997) The self-medication hypothesis of substance use disorders: A reconsideration and recent applications. Harv Rev Psychiatry 4: 231-244.

5. Library of Congress (2005) Immigration: Challenges for new americans.

6. Magana CG, Hovey JD (2003) Psychosocial stressors associated with Mexican migrant farmworkers in the Midwest United 
Citation: Jamil HJ, Niazy A, Jamil MH, et al. (2019) Substance Abuse among Middle Eastern Immigrants and Refugees in Greater Detroit, Michigan, U.S.. Adv Environ Stud 3(1):209-215

States. Journal of Immigrant Health 5: 75-86.

7. NIDA (2015) Nationwide Trends. Retrieved April 24, 2017.

8. Substance abuse and Mental Health Survey Administration (2011) Results from the 2010. National survey on drug use and health: Summary of national findings.

9. Arfken $\mathrm{CL}$, Arnetz BB, Fakhouri M, et al. (2011) Alcohol use among Arab Americans: What is the prevalence? J Immigr Minor Health 4: 713-718.

10. Russel SS (2002) Refugees: Risks and challenges worldwide. Migration information Source - The online journal of migration policy institute.

11. Rehab International (2012) Issues of drug abuse in the middle east.

12. Brown JM, Council CL, Penne MA, et al. (2005) Immigrants and substance use: Findings from the 1999-2001 National surveys on drug use and health. Substance abuse and mental Health Services Administration, Office of Applied Studies.

13. Salas-Wright CP, Vaughn MG, Clark TT, et al. (2014) Substance use disorders among first-and second-generation immigrant adults in the United States: Evidence of an immigrant paradox? J Stud Alcohol Drugs75: 958-967.

14. Bartel Ann P (1989) Where Do the New U.S. Immigrants Live? Journal of Labor Economics 7: 371-391.

15. Chiswick BR, Miller PW (2004) Where Immigrants settle in United States. Journal of Comparative Policy Analysis: Research and Practice.

16. D'Avanzo CE, Frye B, Forman R (1994) Culture, stress and substance use in Cambodian refugee women. J Stud Alcohol 55: 420-426.

17. D'Avanzo CE (1997) Southeast Asians: Asian-Pacific Americans at risk for substance misuse. Substance Use and Misuse 32: 829848.

18. Arfken CL, Jamil H, Arnetz B (2012) Marijuana and non-medical prescription drug use among immigrant Arab Americans. New Iraqi Journal of Medicine 8: 7-13.
19. Padilla AM, Sung H, Nam TV (1993) Attitudes toward alcohol and drinking practices in two Vietnamese samples in Santa Clara County. Horizons of Vietnamese Thought and Expression 2: 53-71.

20. Cervantes RC, Gilbert M, Salgado de Snyder N, et al. (1990-1991) Psychosocial and cognitive correlates of alcohol use in younger adult immigrant and U.S.-born Hispanics. International Journal of Addictions 25: 687-708.

21. Dick DM, Agrawal A, Schuckit MA, et al. (2006) Marital status, alcohol dependence, and GABRA2: Evidence for geneenvironment correlation and interaction. J Stud Alcohol 67: 185194.

22. Merline AC, Schulenberg JE, O'Malley PM, et al. (2008) Substance use in marital dyads: Premarital assortment and change over time. J Stud Alcohol Drugs 69: 352-361.

23. Umberson Debra (1987) Family status and health behaviors: Social control as a dimension of social integration. J Health Soc Behav 306-319.

24. Johnston LD, O'Malley PM, Bachman JG (2003) Monitoring the future national survey results on drug use, 1975-2002: Vol. 1. Secondary School Students.

25. Schnohr Christina (2004) Does educational level influence the effects of smoking, alcohol, physical activity, and obesity on mortality? A prospective population studies. Scand J Public Health 32: 250-256.

26. Degenhardt, Louisa, Wayne Hall (2001) The relationship between tobacco use, substance-use disorders and mental health: Results from the national survey of mental health and well-being. Nicotine Tob Res 3: 225-234.

27. NIDA (2009) Substance abuse among troops, veterans, and their families.

28. Crum RM, Helzer JE, Anthony JC (1993) Level of education and alcohol abuse and dependence in adulthood: A further inquiry. Am J Public Health 83: 830-837.

29. Arfken CL, Broadbridge CL, Jamil H (2014) Immigrant Arab American and Alcohol use; longitudinal study. J Immigr Minor Health 16: 1303.

DOI: $10.36959 / 742 / 218$

Copyright: (C) 2019 Jamil HJ, et al. This is an open-access article distributed under the terms of the Creative Commons Attribution License, which permits unrestricted use, distribution, and reproduction in any medium, provided the original author and source are credited. 